05, 12

\title{
Дискретный энергетический спектр нелинейных спиновых ансамблей в ферримагнетике $\mathrm{K}_{0.4}\left[\mathrm{Cr}(\mathrm{CN})_{6}\right][\mathrm{Mn}(R / S)-p n](R / S)-p n \mathrm{H}_{0.6}$
}

\author{
(C) А.Д. Таланцев, М.В. Кирман, Р.Б. Моргунов \\ Институт проблем химической фризики РАН, \\ Черноголовка, Россия \\ ฯ E-mail: morgunov2005@yandex.ru
}

(Поступила в Редакцию 26 мая 2016 г.)

\begin{abstract}
Обнаружены серии спонтанных скачков намагниченности на фоне непрерывной релаксации магнитного момента молекулярного ферримагнетика в постоянном магнитном поле. Статистическая обработка массива данных демонстрирует наличие, как минимум, двух мод в распределении скачков перемагничивания по их амплитуде. Количество мод в распределении зависит от величины магнитного поля и температуры, характеризует дискретный энергетический спектр нелинейных спиновых ансамблей, возникающих при низких температурах. Непрерывная компонента магнитной релаксации в области $20-50 \mathrm{~K}$ отвечает движению коллективов доменных стенок, а в области $2-10 \mathrm{~K}$ - релаксационным процессам в спин-солитонной решетке.
\end{abstract}

Работа выполнена при финансовой поддержке гранта РФФИ № 15-02-05149.

DOI: $10.21883 /$ FTT.2017.01.43952.221

\section{1. Введение}

В хиральных молекулярных магнетиках сочетание магнитной анизотропии подрешеток, несимметричного и симметричного обменных взаимодействий, а также большой период решетки (> $10 \AA)$ приводят к возникновению необычных нелинейных спиновых структур (ансамблей спинов), отклик которых обнаруживается в аномальных температурных зависимостях намагниченности [1], генерации нелинейных спиновых возбуждений микроволновым магнитным полем [2], бистабильности ферромагнитного резонанса [3], пайерлсовской динамике доменных стенок [4]. Для тонких пленок гелимагнетиков $(\mathrm{MnSi}, \mathrm{FeGe},(\mathrm{FeCo}) \mathrm{Si})$ одним из проявлений образования нелинейной магнитной фазы (конической, геликоидальной или решетки скирмионов) является скачкообразное изменение намагниченности в магнитном поле при достижении его определенного критического значения [5-7].

Скачки намагниченности могут быть стохастической природы. Наиболее распространенными типами нелинейности в неорганических магнетиках являются скачки Баркгаузена, возникающие в случае необратимого смещения доменных стенок при распаде монодоменного состояния или в результате зарождения новых доменов [8]. Разумеется, перемагничивание и скачки намагниченности при движении доменных стенок не исключены и в молекулярных магнетиках, однако о доменах в этих типах материалов практически ничего не известно. В молекулярных магнетиках хаотические скачки намагниченности могут быть вызваны перегруппировкой спинов в ферромагнитные и антиферромагнитные участки линейных цепочек атомов, из которых построен молекулярный кристалл [9]. В недавних работах $[10,11]$ сообщалось об обнаружении стохастических скачков размагничивания в хиральном молекулярном магнетике, где они отвечают нелинейным трансформациям внутренней структуры спиновых солитонов. Примеры возможного теоретического моделирования таких событий представлены в [12]. Аналогичные экспериментальные результаты были получены позднее для хирального гелимагнетика $\mathrm{Cr}_{1 / 3} \mathrm{NbS}_{2}$, в котором дискретность изменения намагниченности была вызвана образованием спин-солитонных решеток [13]. Результаты упомянутых исследований не позволили судить ни о размерах коллективов частиц со спинами, ни о дискретных уровнях энергии и их зависимости от поля и температуры.

Целью настоящей работы является обнаружение статистических закономерностей скачкообразного перемагничивания молекулярного ферримагнетика $\mathrm{K}_{0.4}\left[\mathrm{Cr}(\mathrm{CN})_{6}\right][\mathrm{Mn}(R / S)-p n](R / S)-p n \mathrm{H}_{0.6}$, а также проверка гипотезы о наличии дискретного энергетического спектра нелинейных спиновых ансамблей (солитонов).

\section{2. Методика}

Химический синтез, рентгеноструктурный анализ и экспресс-аттестация магнитных свойств (коэрцитивная сила, температура Кюри) кристаллов $\mathrm{K}_{0.4}\left[\mathrm{Cr}(\mathrm{CN})_{6}\right][\mathrm{Mn}(R / S)-p n](R / S)-p n \mathrm{H}_{0.6}$ были описаны ранее в [14]. Монокристалл представлял собой игольчатую пластинку размером $\sim 1.5 \times 0.5 \times 0.1 \mathrm{~mm}$.

C помощью СКВИД-магнитометра (MPMS 5XL Quantum Design) были выполнены измерения магнитного момента $M$ образцов в режиме постоянного магнитного поля. Абсолютная погрешность измерений магнитного момента составляла $\sim 10^{-7}$ emu. Измерения проводились в диапазоне температур $T=2-50 \mathrm{~K}$, при которых образец находился в магнитоупорядоченном ферримагнитном состоянии (температура Кюри $53 \mathrm{~K}$ ). В процессе измерения температура образца поддержи- 


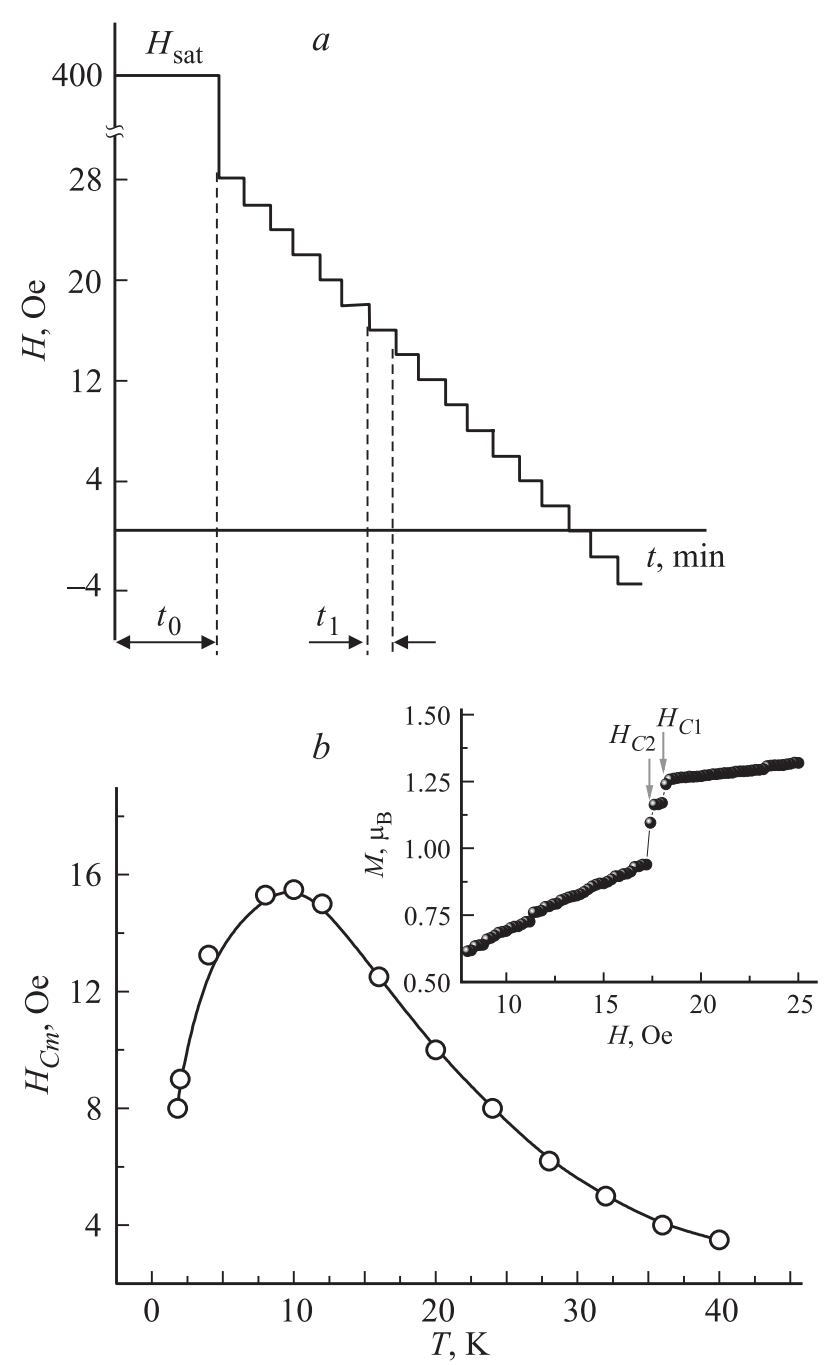

Pис. 1. a) Схема изменения магнитного поля $H(t)$ при измерении полевой зависимости магнитного момента $M(H) . t_{0}$ - время намагничивания образца в поле насыщения $H_{\text {sat }}=400 \mathrm{Oe}$, $t_{1}$ - время измерения магнитного момента. $b$ ) Температурная зависимость среднего значения критического магнитного поля $H_{C m}$, при котором происходит скачок намагниченности максимальной амплитуды. На вставке - зависимость $M(H)$ при переключении поля с шагом $\Delta H=0.2$ Ое при температуре $T=8 \mathrm{~K}$. Стрелками показаны магнитные поля, при которых наблюдаются скачки намагниченности.

валась с точностью $0.1 \mathrm{~K}$. До начала измерений образец был охлажден в нулевом магнитном поле, затем намагничен до насыщения в постоянном магнитном поле $H_{\text {sat }}=400$ Ое в течение $t_{0}=5 \mathrm{~min}$ (см. рис. $1, a$ и схему в верхней части рис. 2,a).

При измерении кривых размагничивания $M(H)$ магнитное поле ступенчато уменьшалось от +28 до -4 Ое с шагом переключения $\Delta H=2-0.2$ Ое (рис. $1, a)$. В серии экспериментов по измерению релаксации магнитного момента $M(t)$ образец находился в постоянном размагничивающем поле $H$ в течение $25 \mathrm{~min}$ (рис. 2,a). Временны́е зависимости магнитного момента $M(t)$ были получены для диапазона полей $H$ от +30 до -50 Oe.

\section{3. Результаты и обсуждение}

3.1. Скачки намагниченности в изменяющемся поле. Полевые зависимости магнитного момента $M(H)$ были измерены в магнитном поле, уменьшающемся от поля насыщения $H_{\text {sat }}=400 \mathrm{Oe}$ с шагом $\Delta H=2-0.2$ Ое при температуре ниже температуры Кюри $T<53 \mathrm{~K}$ (рис. 1). При $T=2 \mathrm{~K}$ магнитный момент ферримагнитного образца в насыщении составляет $M_{\mathrm{sat}}=2 \mu_{\mathrm{B}}\left(\mu_{\mathrm{B}}-\right.$ магнетон Бора $)$, что соответствует антипараллельной ориентации спинов ионов $\mathrm{Mn}^{2+}$ и ионов $\mathrm{Cr}^{3+}$. Магнитное поле было приложено вдоль легкой оси намагничивания. На кривых размагничивания $M(H)$ хирального молекулярного магнетика были обнаружены стохастические скачки магнитного момента, возникающие при достижении критического магнитного поля $H_{C}$ (вставка на рис. $\left.1, b\right)$. Скачки намагниченности воспроизводились на каждой кривой $M(H)$ при температуре $2-50 \mathrm{~K}$. При уменьшении шага переключения поля от 2 до 0.2 Ое (соответственно при уменьшении скорости развертки магнитного поля) было обнаружено, что происходит серия скачков магнитного момента. Наибольшее число скачков наблюдалось при минимальной скорости развертки магнитного поля $0.00125 \mathrm{Oe} / \mathrm{s}$.

С повышением температуры $T>8 \mathrm{~K}$ среднее значение поля скачка наибольшей амплитуды $H_{C m}$ смещается в область низких полей (рис. $1, b)$. При измерении кривых размагничивания $M(H)$ при низких температурах $T<8 \mathrm{~K}$ с интервалом изменения температуры $\Delta T=1 \mathrm{~K}$ было установлено, что зависимость $H_{C m}(T)$ немонотонная: при $T \approx 8 \mathrm{~K}$ наблюдается максимум (рис. $1, b)$.

Для получения статистического распределения скачков магнитного момента и выявления закономерностей их появления была проведена серия экспериментов, состоящая из 20 измерений зависимостей $M(H)$. Исследуемый образец многократно намагничивался и размагничивался в одинаковых условиях (температура, время и поле намагничивания, диапазон размагничивающего поля от +28 до $-4 \mathrm{Oe} \mathrm{c} \mathrm{шагом} \Delta H=0.2 \mathrm{Oe})$. На полученных зависимостях $M(H)$ были выделены скачки магнитного момента, минимальная амплитуда которых составляла $3 \cdot 10^{-6} \mathrm{emu}$. На каждой зависимости $M(H)$ число скачков, их амплитуды $\Delta M$ и магнитные поля $H_{C}$, при которых происходили скачки, принимали значения, не зависящие от предыдущего цикла намагничиванияразмагничивания, т.е. были стохастическими.

Статистический анализ распределения скачков намагниченности по их амплитуде и критическим магнитным полям, в которых они происходят, показывает, что существует закономерность их появления (рис. 3). Скачки магнитного момента можно разбить на моды по изменению амплитуд $\Delta M$ группы скачков. Можно видеть, что скачки наибольшей амплитуды $\left(\Delta M_{3}\right)$ происходят в узком интервале критических магнитных полей $H_{C 3}$, которому соответствует двухмодальное распределение. Интервалам магнитных полей $H_{C 1}, H_{C 2}$ отвечают скачки магнитного момента только одной моды спиновых ан- 

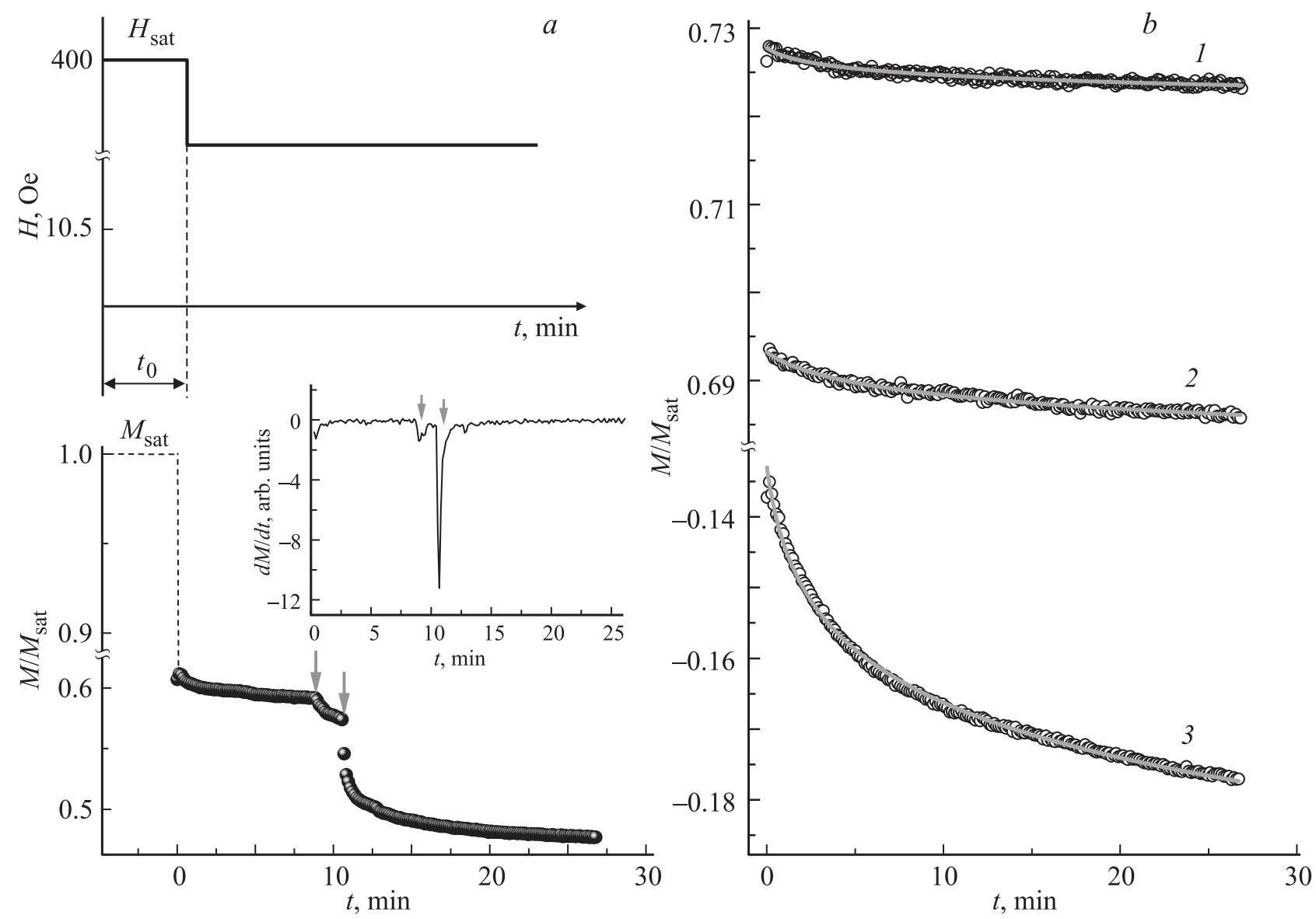

Рис. 2. Зависимость магнитного момента от времени $M(t)$ после включения постоянного поля $H=10.5$ (a) и 30 (1), $20(2)$ и $-18 \mathrm{Oe}(3)(b)$ при $T=2 \mathrm{~K}$. Перед измерением образец был намагничен до насыщения $M_{\text {sat }}$ в магнитном поле $H_{\text {sat }}=400$ Ое в течение времени $t_{0}=5 \mathrm{~min}$. На вставке - производная зависимости $M(t)$ от времени. Стрелками показаны значения времени, при которых происходят скачки намагниченности. Сплошная линия - аппроксимация $M(t)$ по формуле (1).

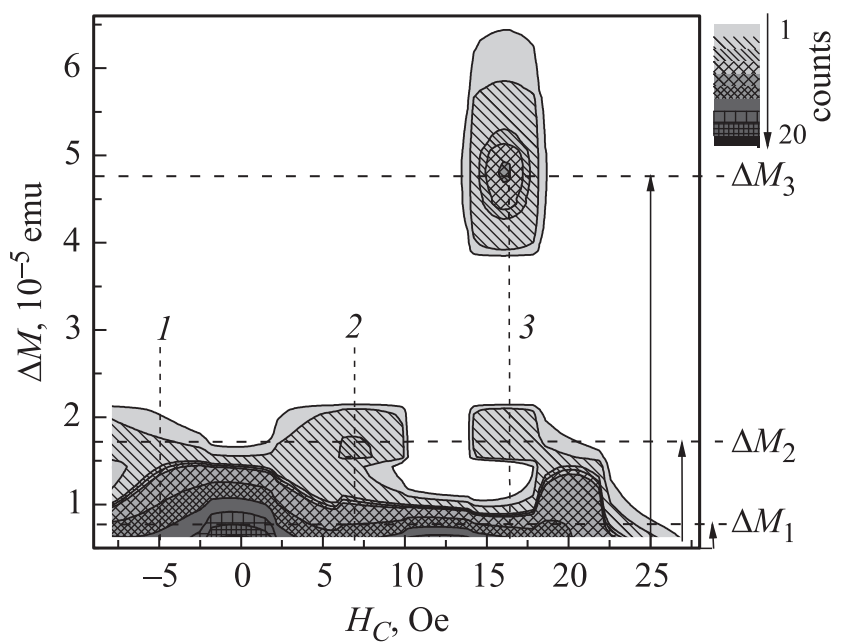

Рис. 3. Распределение амплитуды $\Delta M$ скачков на кривой размагничивания $M(H)$ по значению критического поля $H_{C}$, при достижении которого происходят скачки магнитного момента при $T=8 \mathrm{~K} .1-3-$ состояния спиновых ансамблей, соответствующих значениям критических полей $H_{C 1}, H_{C 2}, H_{C 3}$. самблей. Таким образом, распределение скачков магнитного момента по модам свидетельствует о дискретности энергии групп спинов в кристалле.

3.2. Скачки намагниченности в постоянном магнитном поле. В следующей части работы проводились измерения релаксации момента $M(t)$ в постоянном размагничивающем поле $H$ от +30 до $-50 \mathrm{Oe}$ (рис. 2). До измерения $M(t)$ образец был намагничен до насыщения в магнитном поле $H_{\mathrm{sat}}=400 \mathrm{Oе}$ в течение времени $t_{0}=5 \mathrm{~min}$. В диапазоне полей $H$ от +9 до +10.5 Ое на фоне плавной релаксации $M$ происходят серии резких скачков магнитного момента образца (рис. 2,a). Амплитуда скачка магнитного момента $\Delta M$ определялась по разности значений между магнитными моментами, соответствующих моментам времени начала и завершения минимума на зависимости производной магнитного момента от времени $d M / d t(t)$ (вставка на рис. $2, a)$.

Для статистического анализа скачков была выполнена серия из 20 измерений $M(t)$ в одинаковых условиях: температура, магнитное поле, время измерения. Распределение частоты появления скачков по их амплитуде $\Delta M$ 


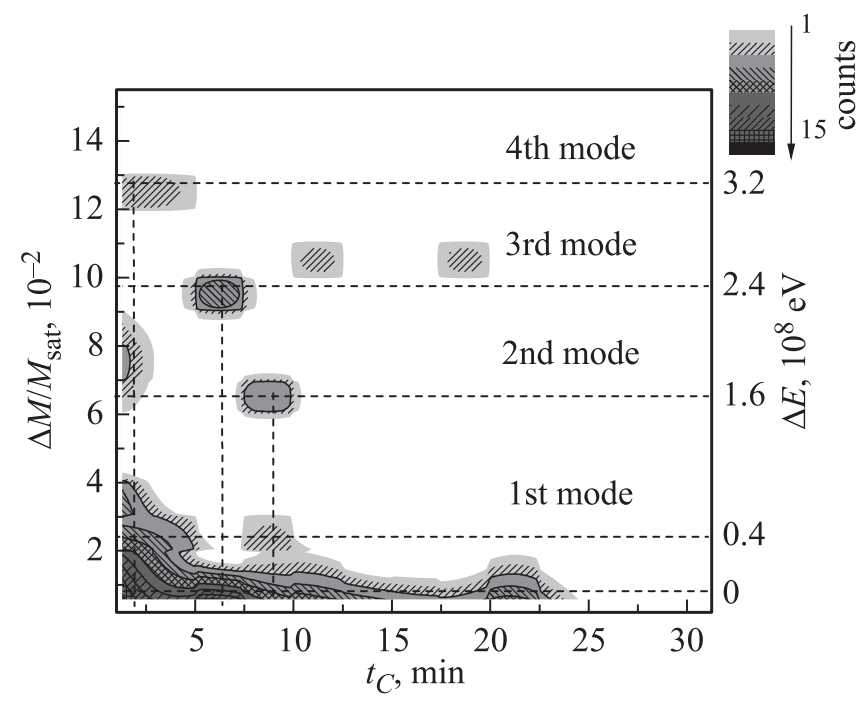

Рис. 4. Статистические распределения амплитуды серии скачков момента по времени $t_{C}$ между включением постоянного магнитного поля $H_{C}=10.5 \mathrm{Oе}$ и временем, спустя которое начинается серия скачков при $T=2 \mathrm{~K}$. Штриховыми линиями обозначены моды скачков, различающихся по амплитуде скачка в момент времени $t$.

и времени $t_{C}$, спустя которое они наблюдались после включения постоянного поля $H$, представлено на рис. 4 . Можно выделить ряд дискретных значений диапазона амплитуд $\Delta M_{i}$, которым могут отвечать энергии спиновых возбуждений $E_{i}$, возникающих во внешнем поле при размагничивании образца, где целое число $i \geq 1$ (рис. 4).

Изменение энергии Зеемана спинового ансамбля при переходе из состояния с энергией $E_{1}$ в состояние с энергией $E_{i}$ будет составлять $\Delta E_{n}=\left(\Delta M_{i}-\Delta M_{1}\right) H$, где $i=n+1, n-$ число переходов между этими состояниями (рис. 4). Таким образом, установлено, что распределение скачков перемагничивания по их амплитуде является мультимодальным. Каждой моде спиновых ансамблей соответствует значение энергии $\Delta E_{n}$, т.е. энергетический спектр мод скачков магнитного момента является дискретным.

3.3. Непрерывная компонента магнитной релаксаци и. Одним из методов изучения динамики доменных стенок является измерение временны́х зависимостей намагниченности. Для большинства магнитотвердых материалов, в частности для молекулярных магнетиков [15], были получены логарифмические зависимости магнитного момента от времени $M(t)$, которые были аппроксимированы выражением

$$
M=M_{0}-S \ln \left(t-t_{01}\right),
$$

где $S$ - магнитная вязкость, $t_{01}$ - время установки поля $H, M_{0}$ - магнитный момент образца после установки поля и начала измерения $M(t)$. Магнитную вязкость можно рассматривать как характеристику энергии активации, необходимой для изменения намагниченности. Например, для перемагничивания с участием доменных стенок энергия активации отвечает высоте потенциального барьера, преодолеваемого движущимися доменными стенками.

Логарифмическая зависимость $M(t)$ свидетельствует о существовании равномерного распределения энергетических барьеров по высоте [16]. С помощью аппроксимации выражением (1) зависимостей $M(t)$ (рис. 2,b), полученных в магнитном поле $H=-H_{\text {coer }}\left(H_{\text {coer }}-\right.$ коэрцитивное поле при температуре $T$ ) были определены значения магнитной вязкости $S$.

В литературе обычно используют значение приведенной вязкости $S_{V}[17,18]$, потому что эта величина не зависит от формы образца: $S_{V}=S / \chi_{\text {irr }}$, где $\chi_{\text {irr }}-$ необратимая магнитная восприимчивость, определяемая выражением

$$
\chi_{\text {irr }}=\frac{\chi}{1+D \chi}
$$

где $\chi=d M / d H-$ угловой коэффициент касательной, проведенной к размагничивающему участку петли магнитного гистерезиса, $D$ - размагничивающий фактор. Для исследуемого образца, имеющего форму игольчатой пластинки, $D \approx 1$.

На рис. 5 представлена температурная зависимость приведенной вязкости $S_{V}(T)$. С повышением температуры магнитная вязкость уменьшается в диапазоне температур $T=2-20 \mathrm{~K}$, а затем при $T>20 \mathrm{~K}$ увеличивается (рис. 5).

Согласно теории закрепления доменных стенок, по значению магнитной вязкости можно оценить активационный объем $V_{0}$, захватывающий область между положениями доменной стенки в соседних минимумах потенциального профиля [19],

$$
S_{V}=k_{\mathrm{B}} T /\left(V_{0} M_{\mathrm{sat}}\right),
$$

где $k_{\mathrm{B}}-$ постоянная Больцмана.

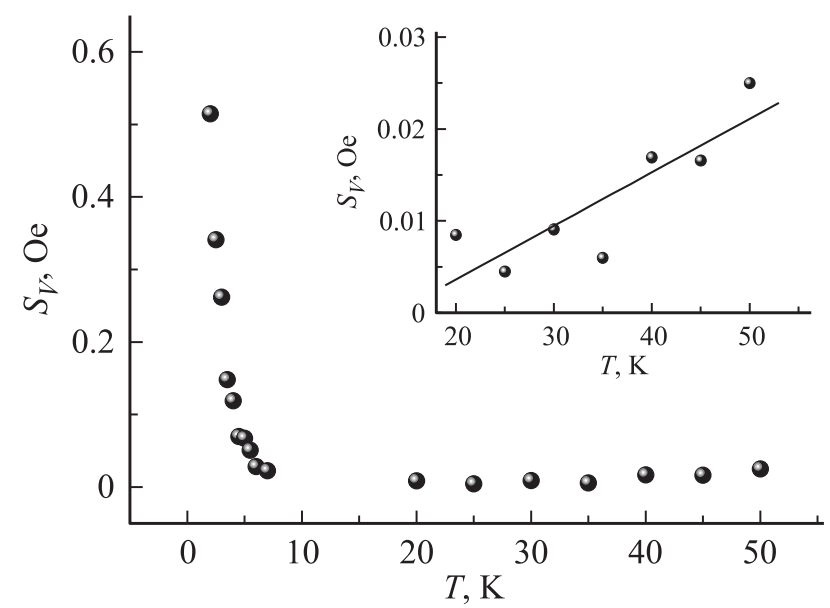

Рис. 5. Температурная зависимость приведенной магнитной вязкости $S_{V}$ в магнитном поле $H=H_{\text {coer }}\left(H_{\text {coer }}-\right.$ коэрцитивное поле при температуре $T$ ). На вставке показана высокотемпературная зависимость приведенной магнитной вязкости $S_{V}$. Сплошной линией показана аппроксимация формулой (3). 
Можно предполагать, что в интервале температур $20-50 \mathrm{~K}$, где зависимость $S_{V}(T)$ линейная (см. вставку на рис. 5), перемагничивание происходит путем движения доменных стенок. С помощью аппроксимации зависимости $S_{V}(T)$ в этом температурном диапазоне выражением (3) получено значение активационного объема $V_{0}=4.4 \cdot 10^{-11} \mathrm{~mm}^{3}$.

Чтобы сравнить это значение активационного объема с полученными для изученных ранее магнитных систем, учтем, что в ферритах и сплавах редкоземельных металлов активационный объем $V_{0} \sim 10 \delta^{3}$ [17], где $\delta-$ ширина доменной стенки. Оценим ширину доменной стенки в исследуемом молекулярном магнетике $\delta$

$$
\delta=\pi \sqrt{\frac{A}{K}}
$$

где $A-$ константа обменного взаимодействия, $K-$ константа анизотропии. С учетом $A=1.6 \cdot 10^{-8} \mathrm{erg} / \mathrm{cm}$, $K=10^{5} \mathrm{erg} / \mathrm{cm}^{3}$ получаем $\delta=12.56 \mathrm{~nm}$. Соответственно активационный объем составит $V_{0} \sim 10^{4} \delta^{3}$. Это значение (на три порядка величины большее, чем в обычных ферромагнетиках) может свидетельствовать о том, что активационный объем следует относить не к одной доменной стенке, а к коллективу стенок.

При $T<10 \mathrm{~K}$ величина $S_{V}$ резко возрастает с понижением температуры (рис. 5), что нельзя объяснить в рамках классической теории движения доменных стенок. Механизм релаксации намагниченности при низких температурах $(T<20 \mathrm{~K})$ может быть объяснен с помощью теории трансформации нелинейных спиновых ансамблей, формирующих описанные выше скачки намагниченности.

\section{4. Заключение}

Обнаружена необычная скачкообразная релаксация намагниченности в магнитохиральных кристаллах $\mathrm{K}_{0.4}\left[\mathrm{Cr}(\mathrm{CN})_{6}\right][\mathrm{Mn}(R / S)-p n](R / S)-p n \mathrm{H}_{0.6}$ в постоянном магнитном поле. Анализ амплитуды скачков, которым соответствует дискретный энергетический спектр, свидетельствует о существовании в кристаллах нелинейных спиновых ансамблей, которые образуются под действием внешнего магнитного поля или температуры. Магнитная вязкость в области высоких температур $(20-50 \mathrm{~K})$ отвечает движению коллективов доменных стенок. В области низких температур $(2-10 \mathrm{~K})$ наблюдается аномальный рост магнитной вязкости с понижением температуры.

\section{Список литературы}

[1] J. Kishine, K. Inoue, Y. Yoshida. Prog. Theor. Phys. Suppl. 159, 82 (2005).

[2] R.B. Morgunov, M.V. Kirman, K. Inoue, Y. Tanimoto, J. Kishine, A.S. Ovchinnikov, O. Kazakova. Phys. Rev. B 77, 184419 (2008).
[3] R.B. Morgunov, F.B. Mushenok, O. Kazakova. Phys. Rev. B 82, 134439 (2010).

[4] F. Mushenok, O. Koplak, R. Morgunov. Eur. Phys. J. B 84, 219 (2011).

[5] A.B. Butenko, A.A. Leonov, U.K. Rößler, A.N. Bogdanov. Phys. Rev. B 82, 052403 (2010).

[6] A. Karhu, U.K. Rößler, A.N. Bogdanov, S. Kahwaji, B.J. Kirby, H. Fritzsche, M.D. Robertson, C.F. Majkrzak, T.L. Monchesky. Phys. Rev. B 85, 094429 (2012).

[7] M.N. Wilson, E.A. Karhu, D.P. Lake, A.S. Quigley, S. Meynell, A.N. Bogdanov, H. Fritzsche, U.K. Rößler, T.L. Monchesky. Phys. Rev. B 88, 214420 (2013).

[8] В.М. Рудяк. УФН 101, 429 (1970).

[9] A.S. Boyarchenkov, I.G. Bostrem, A.S. Ovchinnikov. Phys. Rev. B 76, 224410 (2007).

[10] М.В. Кирман, А.Д. Таланцев, О.В. Коплак, Р.Б. Моргунов. Письма в ЖЭТФ 101, 433 (2015).

[11] Р.Б. Моргунов, М.В. Кирман, А.Д. Таланцев. ФТТ 57, 1498 (2015).

[12] J. Kishine, I.G. Bostrem, A.S. Ovchinnikov, Vl.E. Sinitsyn. Phys. Rev. B 89, 014419 (2014).

[13] K. Tsuruta, M. Mito, Y. Kousaka, J. Akimitsu, J. Kishine, Y. Togawa, H. Ohsumi, K. Inoue. J. Phys. Soc. Jpn. 85, 013707 (2016).

[14] K. Inoue, H. Imai, P.S. Ghalsasi, K. Kikuchi, M. Ohba, H. Okawa, J.V. Yakhmi. Angew. Chem. Int. Ed. 40, 4242 (2001).

[15] M. Sendek, K. Csach, V. Kavečanský, M. Lukáčová, M. Maryško, Z. Mitróová, A. Zentko. Phys. Status Solidi A 196, 225 (2003).

[16] D.K. Lottis, E.D. Dahlberg, J.A. Christner, J.I. Lee, R.L. Peterson, R.M. White. J. Appl. Phys. 63, 2920 (1988).

[17] D. Givord, Q. Lu, M.F. Rossignol, P. Tenaud, T. Viadieu. J. Magn. Magn. Mater. 83, 183 (1990).

[18] C.K. Mylvaganam, P. Gaunt. Phil. Mag. B 44, 581 (1981).

[19] P. Gaunt. J. Appl. Phys. 59, 4129 (1986). 